\title{
Conformational and Packing Stability of Crystalline Polymers. VII. A Method for the Minimization of Conformational and Packing Energies of Crystalline Polymers
}

\author{
Hiroshi Kusanagi, Hiroyuki Tadokoro, and Yozo Chatani \\ Department of Polymer Science, Faculty of Science, Osaka University, \\ Toyonaka, Osaka 560, Japan.
}

(Received December 6, 1976)

\begin{abstract}
An energy minimization method for seeking stable crystal structure in terms of the nonbonded repulsive energy alone was derived. The variable parameters are molecular positions and their internal coordinates; the unit cell dimensions and the space group are fixed to those obtained by X-ray analysis. By starting from the crystal structure models composed of uniform helical chains for polyisobutylene, poly(ethylene oxide), and isotactic polypropylene, the present method gave energetically stable structures which are very close to those determined by X-ray analyses. Furthermore, a constrained least-squares method was derived, in which the intra- and intermolecular repulsive energies were taken into account in addition to the differences of the observed and calculated structure factors. This constrained least-squares refinement was successfully applied to the crystal structure analysis of isotactic poly(4-methyl-1-pentene).

KEY WORDS Energy Minimization / X-Ray Analysis / Crystal Structure / Polyisobutylene / Poly(ethylene oxide) / Isotactic Polypropylene / Isotactic Poly(4-methyl-1-pentene) / Repulsive Energy /
\end{abstract}

In the previous papers of this series, several methods $^{1,2}$ for setting up molecular models were derived for X-ray structure analyses of polymers, based upon the intramolecular interaction energy calculations. These methods have been applied successfully to the structure analyses of several polymers. $^{3,4}$ However, the crystal structure models obtained by packing these molecular models in the unit cell are not always satisfactory as the models for carrying out the analysis, and some cases ${ }^{5}$ have been found in which the influence of intermolecular interactions on the flexible molecular chains of polymers cannot be neglected. It is, therefore, quite important to seek more suitable crystal structure models by calculating the potential energy due to both the intra- and intermolecular interactions.

Although there have been papers concerning the energy minimization of three-dimensional crystals of high polymers by McGuire, et al., ${ }^{6}$ and Hermans, et al., ${ }^{7}$ their methods require a very large number of computations, because of the optimization problem of a large number of parameters; the former uses line searches and quadratic interpolations and the latter the Newton-Raphson method.

In the present work, the internal coordinates (bond lengths, bond angles, and internal rotation angles) were used as the parameters and the quadratic potential functions ${ }^{8}$ were introduced as the potentials. Thereby we derived a new method, with fewer calculations, in which the energy minimization was carried out by the leastsquares method. Moreover, a constrained leastsquares refinement method was derived, in which the intra- and intermolecular repulsive energies were taken into account in addition to the differences of the observed and calculated structure factors. This constrained least-squares refinement was applied to the crystal structure analysis of isotactic poly(4-methyl-1-pentene).

\section{METHOD AND ASSUMPTION}

\section{Potential Functions}

In the present calculation, only the repulsive 
Table I. Parabolic potential constants for calculating nonbonded interactions ${ }^{\mathrm{a}}$

\begin{tabular}{|c|c|c|c|c|c|c|c|c|}
\hline \multirow{2}{*}{ Atom or group } & \multicolumn{4}{|c|}{ Intramolecular } & \multicolumn{4}{|c|}{ Intermolecular } \\
\hline & $r_{1}^{\circ}, \mathrm{A} k_{1}$ & mdyn/A & $r_{2}^{\circ}, \mathrm{A}$ & $k_{2}, \operatorname{mdyn} / \mathbf{A}$ & $r_{1}^{\circ}, \mathrm{A}$ & $k_{1}, \operatorname{mdyn} / \mathbf{A}$ & $r_{2}^{\circ}, \mathrm{A}$ & $k_{2}, \operatorname{mdyn} / \mathrm{A}$ \\
\hline $\mathbf{H} \cdots \mathbf{H}$ & 2.0 & 0.074 & 2.3 & 0.0074 & 2.2 & 0.102 & 2.6 & 0.0072 \\
\hline $\mathrm{C} \cdots \mathrm{C}$ & 2.8 & 0.047 & 3.2 & 0.0049 & 3.1 & 0.037 & 3.6 & 0.0033 \\
\hline C...C (carbonyl) & 2.8 & 0.075 & 3.2 & 0.0077 & 3.1 & 0.058 & 3.6 & 0.0052 \\
\hline C...C (aromatic) & 3.0 & 0.032 & 3.4 & 0.0037 & 3.3 & 0.026 & 3.8 & 0.0027 \\
\hline O...O (ether) & 2.5 & 0.077 & 2.9 & 0.0067 & 2.8 & 0.062 & 3.3 & 0.0043 \\
\hline O...O (carbonyl) & 2.6 & 0.063 & 3.0 & 0.0051 & 2.9 & 0.054 & 3.4 & 0.0039 \\
\hline $\mathrm{CH}_{3} \cdots \mathrm{CH}_{3}$ & 3.4 & 0.039 & 3.8 & 0.0046 & 3.7 & 0.040 & 4.2 & 0.0038 \\
\hline $\mathrm{CH}_{2} \cdots \mathrm{CH}_{2}$ & 3.4 & 0.025 & 3.8 & 0.0031 & 3.7 & 0.025 & 4.2 & 0.0028 \\
\hline $\mathrm{CH} \ldots \mathrm{CH}$ & 3.4 & 0.015 & 3.8 & 0.0019 & 3.7 & 0.014 & 4.2 & 0.0016 \\
\hline
\end{tabular}

- The subscripts 1 and 2 indicate the ranges from potential minimum to about $0.5 \mathrm{kcal} / \mathrm{mol}$ and from $0.5 \mathrm{kcal} / \mathrm{mol}$ to about $3.0 \mathrm{kcal} / \mathrm{mol}$, respectively.
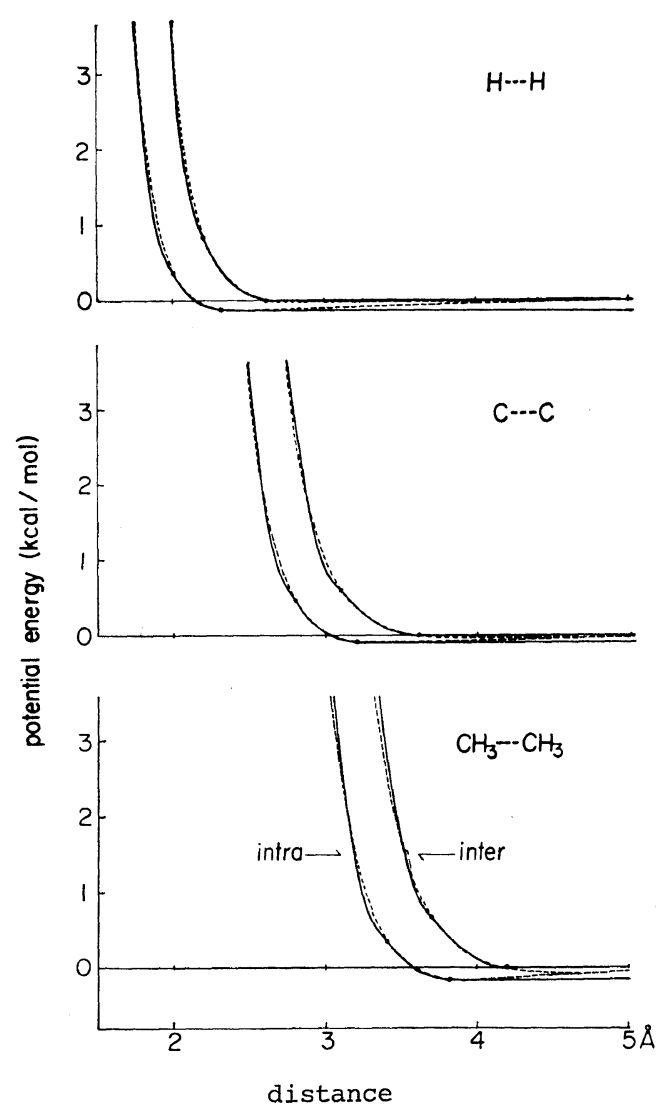

Figure 1. Nonbonded interatomic potential curves for $\mathrm{H} \cdots \mathrm{H}, \mathrm{C} \cdots \mathrm{C}$, and $\mathrm{CH}_{3} \cdots \mathrm{CH}_{3}$ interactions: solid line, parabolic repulsive potential; broken line, Lennard-Jones 6-12 potential. force between nonbonded atoms was taken into account. The nonbonded interatomic potential energy $U\left(r_{i j}\right)$ is approximated by a parabolic function of eq 1 , according to the procedure developed by Williams. ${ }^{8}$

$$
U\left(r_{i j}\right) \begin{cases}=\frac{1}{2} k_{i j}\left(r_{i j}^{\circ}-r_{i j}\right)^{2}, & \text { for } r_{i j}^{\circ} \geqq r_{i j} \\ =0, & \text { for } r_{i j}^{\circ}<r_{i j}\end{cases}
$$

Here $r_{i j}^{\circ}$ and $k_{i j}$ are the van der Waals distance and the force constant, respectively, between the $i$ th and $j$ th atoms. The constants were determined by trial-and-error procedures so that the parabolic function curves would fit well to the Lennard-Jones 6-12 potential function curves, i.e., the sets $I$ and $V$ derived for the intra- and intermolecular interactions in this laboratory, ${ }^{9}$ in the range from the minimum to about $3 \mathrm{kcal} / \mathrm{mol}$. For the interaction of the pairs $\mathrm{H} \cdots \mathrm{C}, \mathrm{H} \cdots \mathrm{CH}_{3}$, etc., the constants were estimated by the empirical combining law. ${ }^{10}$ The parabolic constants used are listed in Table I. The parabolic potential curves are shown in Figure 1, and compared with the 6-12 potential curves.

\section{Energy Minimization Procedure}

For the first approximation, the conformational and packing energy $E$ of a crystal is represented by the sum of the nonbonded atomic potential energies as follows:

$$
E=\frac{1}{2} \sum_{i, j} U\left(r_{i j}\right)=\frac{1}{4} \sum_{i, j} k_{i j}\left(r_{i j}^{\circ}-r_{i j}\right)^{2}
$$


where $r_{i j}$ is any nonbonded interatomic distance in the crystal and can be represented as a function of the structural parameters $u_{n}(n=1,2, \ldots$, $N)$ as described below. The intramolecular interactions are summed over all pairwise nonbonded distances in a crystallographic asymmetric unit and also over all nonbonded distances between the asymmetric unit and successive asymmetric units, except for the pairs which do not depend on the conformation. For the intermolecular interactions, the pairs are summed up from the asymmetric unit to surrounding molecules in the crystal. A stable crystal structure model can be obtained by minimizing $E$. In order to maintain an acceptable stereochemistry at the junction of successive asymmetric units during the minimization, constraining conditions $G_{h}=0(h=1,2, \ldots, H, H<N)$ were introduced with the Lagrange undetermined multipliers $\lambda_{h}{ }^{11,12}$ A stable conformational and packing geometry in the crystal structure is obtained by minimizing $\Phi$ in terms of the leastsquares procedure.

$$
\Phi=E+\sum_{h=1}^{H} \lambda_{h} G_{h}
$$

The normal equations are obtained by using a first order Taylor's series expansion of eq 4:

$$
\begin{aligned}
\Phi= & \frac{1}{4} \sum_{i, j} k_{i j}\left[\Delta r_{i j}\left(u_{n}\right)-\sum_{n=1}^{H} \Delta u_{n} \frac{\partial r_{i j}\left(u_{n}\right)}{\partial u_{n}}\right]^{2} \\
& +\sum_{h=1}^{H} \lambda_{h}\left[G_{h}+\sum_{n=1}^{N} \Delta u_{n} \frac{\partial G_{h}}{\partial u_{n}}\right]
\end{aligned}
$$

where

$$
\Delta r_{i j}=r_{i j}^{\circ}-r_{i j}
$$

These normal equations may be written in matrix notation and solved in the same way as those given in ref 11 .

\section{Interatomic Distances and Their Derivatives by Internal Coordinates}

For solving the normal equations, it is necessary to represent analytically the interatomic distance $r_{i j}$ and the derivatives of $r_{i j}$ and $G_{h}$ with respect to the parameters $u_{n}$. By using the unit cell dimensions ( $a, b, c, \alpha, \beta$, and $\gamma$ ) and the space group obtained by $X$-ray analysis, a set of parameters $u_{n}$ is limited to a crystallographic asymmetric unit. Thereby this minimization method may be applicable to any space group.

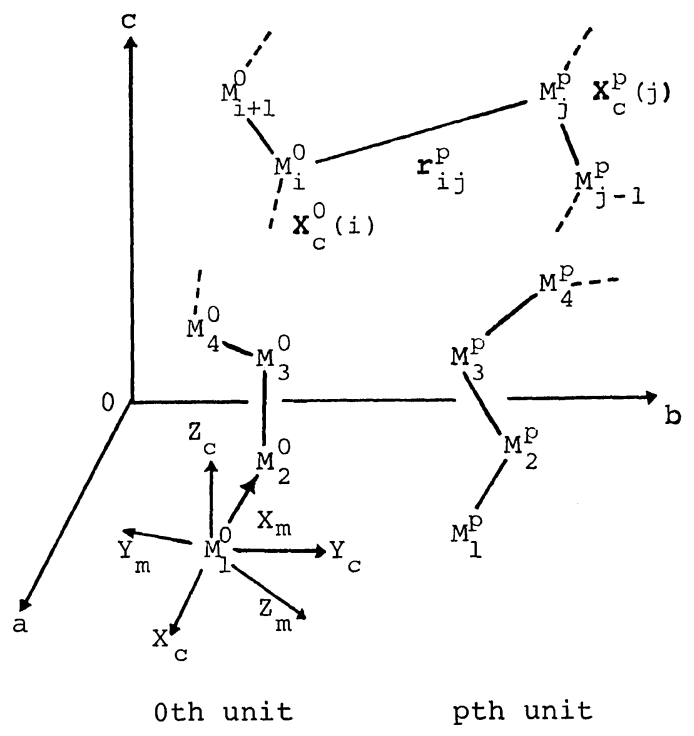

Figure 2. Interatomic distance $r_{i j}^{p}$ in the cartesian coordinate system $\boldsymbol{X}_{\mathrm{c}} . \quad \boldsymbol{X}_{\mathrm{c}}{ }^{p}(j)$ is the set of coordinates of the $j$ th atom in the $p$ th unit.

As shown in Figure 2, a right-handed cartesian coordinate system $\boldsymbol{X}_{\mathrm{c}}$ is defined as a basic coordinate system in the crystal. Here the first atom of the 0th asymmetric unit is taken as the origin, the $Z_{\mathrm{c}}$ axis is parallel to the crystal axis $c$, and the $Y_{\mathrm{c}}$ axis is parallel to the $b c$ plane. The distance $r_{i j}^{p}$ between the $i$ th atom in the 0 th asymmetric unit and the $j$ th atom in the $p$ th asymmetric unit is given by the following equation:

$$
\begin{aligned}
\boldsymbol{r}_{i j}^{p} & =\left|\boldsymbol{r}_{i j}^{p}\right|=\left|\boldsymbol{X}_{\mathrm{c}}{ }^{0}(i)-\boldsymbol{X}_{\mathrm{c}}{ }^{p}(j)\right| \\
& =\left|\boldsymbol{X}_{\mathrm{c}}{ }^{0}(i)-\mathbf{R}^{p} \boldsymbol{X}_{\mathrm{c}}{ }^{0}(j)-\boldsymbol{D}^{p}\right|
\end{aligned}
$$

where $\boldsymbol{X}_{\mathrm{c}}{ }^{0}(i)$ and $\boldsymbol{X}_{\mathrm{c}}{ }^{p}(j)$ are the coordinates of the two atoms, respectively, and $\mathbf{R}^{p}$ and $D^{p}$ are the rotation matrix and the translation column vector which are defined below. The cartesian coordinate system $\boldsymbol{X}_{\mathrm{c}}$ is related to the fractional coordinate system $\boldsymbol{x}$ by the following equation:

$$
\boldsymbol{x}=\mathbf{C} \boldsymbol{X}_{\mathrm{c}}+\boldsymbol{T}
$$

where $T$ is the fractional coordinate vector of the origin atom $\mathbf{M}_{1}{ }^{0}$, and the matrix $\mathbf{C}$ is the transformation matrix* between the coordinate

* The definition of $\mathbf{C}$ in the present paper is the same as those given in ref 11 and 12. The matrix C corresponds to the inverse of the $\mathbf{C}$ in Williams' paper. $^{8}$ 
systems $\boldsymbol{X}_{\mathbf{c}}$ and $\boldsymbol{x}$. The elements of $\mathbf{C}$ are

$$
\mathbf{C}=\left[\begin{array}{ccc}
\left(a \sin \beta \sin \gamma^{*}\right)^{-1} & 0 & 0 \\
\left(b \sin \alpha \tan \gamma^{*}\right)^{-1} & (b \sin \alpha)^{-1} & 0 \\
\frac{-\left(\sin \beta \cos \gamma^{*}+\tan \alpha \cos \beta\right)}{c \tan \alpha \sin \beta \sin \gamma^{*}} & -(c \tan \alpha)^{-1} & 1 / c
\end{array}\right]
$$

The transformations in the fractional coordinate system between the aforementioned two atoms are given by

$$
\boldsymbol{x}^{p}(j)=\mathbf{S}^{p} \boldsymbol{x}^{0}(j)+\boldsymbol{L}^{p}
$$

The matrix $\mathbf{S}$ and vector $\boldsymbol{L}$ are obtained from "Coordinates of Equivalent Positions" taken from the International Tables for X-ray Crystallography. Here

$$
\mathbf{R}^{p}=\mathbf{C}^{-1} \mathbf{S}^{p} \mathbf{C} \quad \text { and } \quad D^{p}=\mathbf{C}^{-1} L^{p}
$$

The $r_{i j}^{p}$ is expanded into the components in the coordinate system $X_{\mathrm{c}}$ in the following form:

$$
r_{i j}^{p}=\left[\left(r_{i j}^{p}\right)_{1}{ }^{2}+\left(r_{i j}^{p}\right)_{2}{ }^{2}+\left(r_{i j}^{p}\right)_{3}{ }^{2}\right]^{1 / 2}
$$

where

$$
\begin{aligned}
\left(r_{i j}^{p}\right)_{\rho}= & X_{\mathrm{c} \rho}^{0}(i)-R_{\rho 1}^{p} X_{\mathrm{c} 1}^{0}(j)-R_{\rho 2}^{p} X_{\mathrm{c} 2}^{0}(j) \\
& -R_{\rho 3}^{p} X_{\mathrm{c} 3}^{0}(j)-D_{\rho}{ }^{p}
\end{aligned}
$$

and $\rho=1,2,3$, correspond to $X_{\mathrm{c}}, Y_{\mathrm{c}}$, and $Z_{\mathrm{c}}$, respectively.

Thus the derivatives of the distance $r_{i j}^{p}$ with respect to the parameters $u_{n}$ are given in the simple formulas of eq 6 :

$$
\begin{gathered}
\frac{\partial r_{i j}^{p}}{\partial u_{n}}=\frac{1}{r_{i j}^{p}}\left[r_{i j 1}^{p} \frac{\partial\left(r_{i j}^{p}\right)_{1}}{\partial u_{n}}+r_{i j 2}^{p} \frac{\partial\left(r_{i j}^{p}\right)_{2}}{\partial u_{n}}+r_{i j 3}^{p} \frac{\partial\left(r_{i j}^{p}\right)_{3}}{\partial u_{n}}\right] \\
\frac{\partial\left(r_{i j}^{p}\right)_{\rho}}{\partial u_{n}}= \\
=\frac{\partial X_{\mathrm{c} 1}^{0}(i)}{\partial u_{n}}-R_{\rho 1}^{p} \frac{\partial X_{\mathrm{c} 1}^{0}(j)}{\partial u_{n}}-R_{\rho 2}^{p} \frac{\partial X_{\mathrm{c} 2}^{0}(j)}{\partial u_{n}} \\
-R_{\rho 3}^{p} \frac{\partial X_{\mathrm{c} 3}^{0}(j)}{\partial u_{n}}
\end{gathered}
$$

The constraining condition $G_{h}=0$ is given by the following simple equation: ${ }^{12}$

$$
G_{h}=\left|\boldsymbol{X}_{\mathrm{c}}{ }^{0}(i)-\mathbf{R}^{p} \boldsymbol{X}_{\mathrm{c}}^{0}(j)-D^{p}\right|^{2}-d^{2}=0
$$

where $d$ is the distance to be constrained, and also the derivatives of the constraining conditions are the same as those given in ref 12 .

The coordinates $\boldsymbol{X}_{\mathrm{c}}{ }^{0}(i)$ are expressed by eq 7 , in terms of the other right-handed cartesian coordinate system $\boldsymbol{X}_{\mathrm{m}}$, in which the origin coincides with that of the coordinate system $\boldsymbol{X}_{\mathrm{c}}$, the second atom $\mathrm{M}_{2}{ }^{0}$ lies on the $X_{\mathrm{m}}$ axis, and the other axes are chosen arbitrarily:

$$
\boldsymbol{X}_{\mathrm{c}}{ }^{0}(i)=\mathbf{Q} \boldsymbol{X}_{\mathrm{m}}(i)
$$

where $\boldsymbol{X}_{\mathrm{m}}(i)$ is the set of coordinates of the $i$ th atom in the coordinate system $\boldsymbol{X}_{\mathrm{m}}$, and $\mathbf{Q}$ is the Eulerian matrix between the coordinate systems $\boldsymbol{X}_{\mathrm{c}}$ and $\boldsymbol{X}_{\mathrm{m}}$, and

where

$$
\boldsymbol{X}_{\mathrm{m}}(i)=\boldsymbol{B}_{12}+\mathbf{A}_{12} \boldsymbol{B}_{23}+\cdots+\mathbf{A}_{12} \boldsymbol{B}_{23} \mathbf{A}_{23} \cdots \mathbf{A}_{\boldsymbol{i}-2, i-1} \boldsymbol{B}_{i-1, i}
$$

$$
\begin{aligned}
& \boldsymbol{X}_{\mathrm{m}}(1)=\left[\begin{array}{l}
0 \\
0 \\
0
\end{array}\right], \quad \boldsymbol{X}_{\mathrm{m}}(2)=\left[\begin{array}{c}
b_{12} \\
0 \\
0
\end{array}\right], \\
& \mathbf{A}_{i-1, i}=\left[\begin{array}{ccc}
-\cos \phi_{i} & -\sin \phi_{i} & \\
\sin \phi_{i} \cos \tau_{i-1, i} & -\cos \phi_{i} \cos \tau_{i-1, i} & -\sin \tau_{i-1, i} \\
\sin \phi_{i} \sin \tau_{i-1, i} & -\cos \phi_{i} \sin \tau_{i-1, i} & \cos \tau_{i-1, i}
\end{array}\right], \\
& \boldsymbol{B}_{i-1, i}=\left[\begin{array}{c}
b_{i-1, i} \\
0 \\
0
\end{array}\right]
\end{aligned}
$$

Here $b_{i-1, i}, \phi_{i}$, and $\tau_{i-1, i}$ are the internal coordinates defined in ref 12 . Using these equations, the derivatives $\partial X_{\mathrm{c}}(i) / \partial u_{n}$ contained in eq 6 are calculated according to the procedure given by Scheringer ${ }^{13}$ and Sugeta-Miyazawa. ${ }^{14}$

Consequently, the variable parameters $u_{n}$ become as follows: (a) the fractional coordinates of the atom chosen as the origin of the coordinate system $\boldsymbol{X}_{\mathrm{m}}$ (molecular position parameters), (b) the Eulerian angles $\theta, \phi$, and $\chi$ (molecular orientation parameters), and (c) the internal rotation angles in an asymmetric unit (molecular conformation parameters). 
A Least-Squares Refinement for X-Ray Analysis with Energy Constraint

Since the present energy minimization method adopted the parabolic potential function of eq 1 , i.e., the square of the difference between the van der Waals distance $r^{\circ}$ and the interatomic distance $r$, the energy terms can be combined as the constraining condition with the leastsquares refinement procedure for X-ray analysis. The minimization function $\Theta$ is given by eq 8 .

$$
\Theta=\sum_{m=1}^{M}\left|\sqrt{I_{\mathrm{o}}(m)}-\sqrt{I_{\mathrm{c}}(m)}\right|^{2}+\omega \Phi
$$

where $\sqrt{I_{\mathrm{o}}(m)}$ and $\sqrt{I_{\mathrm{c}}(m)}$ are the square roots of the $m$ th observed and calculated diffraction intensities, respectively, and $\Phi$ is the energy term in eq 3. The coefficient $\omega$ indicates the weight of energy constraint to the least-squares refinement of the structure factors.

The programs were written in Fortran Language. One further device was taken into account in practice. Since the starting models in many cases have very short interatomic distances, the first order approximation of the Taylor's series expansion of eq 4 does not hold in adequate accuracy. In such cases the constraining condition $G_{h}=0$ cannot always be maintained in the minimization process. In order to overcome this difficulty, we have kept the shifts of the atomic coordinates to be obtained from computation smaller than $0.1 \mathrm{~A}$ per cycle.

Computations were made by the NEAC 2200 Model 700 digital computer in this university.

\section{APPLICATION AND RESULTS}

For polyisobutylene (PIB) and poly(ethylene oxide) (PEO) the molecular conformations in the crystals deform significantly from $(8 / 3)^{15}$ and $(7 / 2)^{16}$ uniform helices, respectively, while the molecular conformation of isotactic polypropylene (IPP) keeps an almost exact (3/1) helical symmetry in the crystal. ${ }^{17-19}$ In order to examine the conformational and packing stability of polymer chains in crystals and also to clarify the validity of this energy minimization method, the most stable conformation and the molecular packing were calculated for these polymers by using the present energy minimization method, and the results were compared with the structures determined by X-ray analyses.

As a measure of the discrepancy between the atomic coordinates obtained by the energy minimization method and the X-ray analysis, an

Table II. Crystallographic data for calculations

\begin{tabular}{lcc}
\hline Polymer & Molecular conformation & Crystal system, space group, and unit cell dimensions ${ }^{\mathrm{a}}$ \\
\hline PIB $^{15}$ & $(8 / 3)$ Helix & Orthorhombic, $\mathrm{P} 2_{1} 2_{1} 2_{1}\left(\mathrm{D}_{2}{ }^{4}\right), a=6.88 \mathrm{~A}, b=11.91 \mathrm{~A}, c=18.60 \mathrm{~A}$ \\
PEO $^{16}$ & $(7 / 2)$ Helix & Monoclinic, $\mathrm{P} 2_{1} / \mathrm{a}\left(\mathrm{C}_{2 \mathrm{~h}}^{5}\right), a=8.16 \mathrm{~A}, b=12.99 \mathrm{~A}, c=19.30 \mathrm{~A}, \beta=126.1^{\circ}$ \\
$\mathrm{IPP}^{17-19}$ & $(3 / 1) \mathrm{Helix}^{\mathrm{b}}$ & Monoclinic, $\mathrm{P} 2_{1} / \mathrm{c}\left(\mathrm{C}_{2 \mathrm{~h}}^{5}\right), a=6.63 \mathrm{~A}, b=20.78 \mathrm{~A}, c=6.504 \mathrm{~A}, \beta=99.5^{\circ}$ \\
\hline
\end{tabular}

a Fiber axis coincides with $c$ axis.

b The structure by Mencik $^{18}$ was used for IPP, but the statistical disorder was ignored for simplicity.

Table III. Definition of internal rotation angles in a crystallographic asymmetric unit considering space groups for PIB, PEO, and IPP

\begin{tabular}{|c|c|c|c|}
\hline Polymer & $\begin{array}{l}\text { Space } \\
\text { group }\end{array}$ & Numbering of internal rotation angles & $\begin{array}{c}\text { Number of } \\
\text { monomeric } \\
\text { units in } \\
\text { asymmetric } \\
\text { unit }\end{array}$ \\
\hline PIB $^{15}$ & $\mathrm{P} 2{ }_{1} 2_{1} 2_{1}$ & $\begin{array}{c}-\mathrm{CH}_{2}-\mathrm{CM}_{2}-\mathrm{CH}_{2}-\mathrm{CM}_{2}-\mathrm{CH}_{2}-\mathrm{CM}_{2}-\mathrm{CH}_{2}-\mathrm{CM}_{2}-\mathrm{CH}_{2}- \\
\tau_{1} \quad \tau_{2} \quad \tau_{3} \quad \tau_{4} \quad \tau_{5} \quad \tau_{6} \quad \tau_{7} \tau_{8}\end{array}$ & 4 \\
\hline $\mathrm{PEO}^{16}$ & $\mathbf{P} 2{ }_{1} / \mathbf{a}$ & Only translation $\left.+\mathrm{O}-\mathrm{CH}_{2}-\mathrm{CH}_{2}-\mathrm{O}-\mathrm{CH}_{2}-\mathrm{CH}_{2}-\cdots-\mathrm{CH}_{2}-\mathrm{CH}_{2}\right\}-\mathrm{O}-$ & 7 \\
\hline IPP $17-19$ & $\mathrm{P} 2{ }_{1} / \mathrm{c}$ & 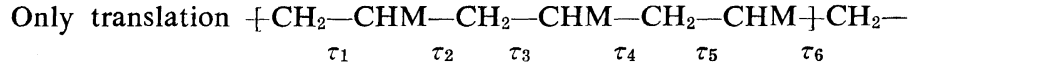 & 3 \\
\hline
\end{tabular}




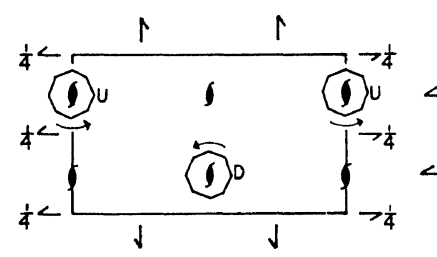

(a)

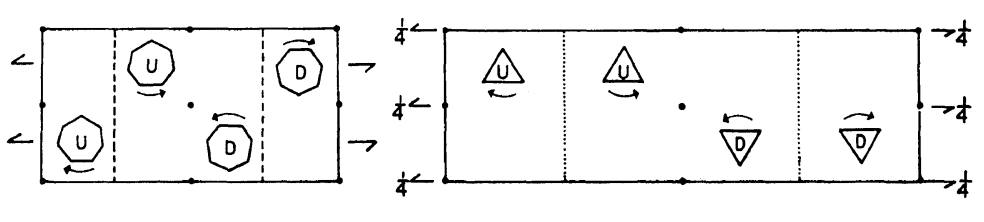

(b) (c)

Figure 3. The schemes of molecular packing in the unit cell under the space group projected along the $c$ axis: (a), PIB; (b), PEO; (c), IPP. U and D denote upward and downward helices, respectively.

average deviation $\bar{d}$ was calculated for each structure: ${ }^{20}$

$$
\bar{d}=\left(\sum_{i=1}^{3 N} \Delta_{i}{ }^{2} / 3 N\right)^{1 / 2}
$$

where $N$ is the number of atoms in an asymmetric unit and $\Delta_{i}$ is the difference between the corresponding atomic coordinates obtained by the energy minimization method and by X-ray analysis.

The crystallographic data used for the calculations are listed in Table II. The bond lengths and bond angles used are as follows: bond lengths, $\mathrm{C}-\mathrm{C}=\mathrm{C}-\mathrm{CH}_{2}=\mathrm{C}-\mathrm{CH}_{3}=1.54 \mathrm{~A}$ and $\mathrm{C}-\mathrm{O}=1.43 \mathrm{~A}$; bond angles, $\angle \mathrm{CH}_{2}-\mathrm{C}-\mathrm{CH}_{2}=$ $110^{\circ}$ and $\angle \mathrm{C}-\mathrm{CH}_{2}-\mathrm{C}=128^{\circ}$ for PIB, $\angle \mathrm{OCO}=$ $110^{\circ}$ and $\angle \mathrm{OCC}=112^{\circ}$ for PEO, $\angle \mathrm{CCC}=114^{\circ}$ for IPP (skeletal chain), and other bond angles= $109.5^{\circ}$. The methyl and methylene groups were considered as a unit in the energy calculation. The number of independent structural parameters (internal rotation angles) is connected with the symmetry of the crystal structure. Table III gives the numbering of the internal rotation angles considering the space group for PIB, PEO, and IPP. The schemes of the molecular packing are also shown in Figure 3.

\section{Polyisobutylene}

Since the PIB molecule in the orthorhombic unit cell contains eight monomeric units in the fiber identity period and can possess only a twofold screw symmetry, the crystallographic asymmetric unit must consist of four successive monomeric units, as shown in Table III. In this minimization method the variable parameters are the three fractional coordinates $x y z$ of the carbon atom $\mathrm{C}(1)$ chosen as the origin of the coordinate system $\boldsymbol{X}_{\mathrm{m}}$ fixed on the molecule,

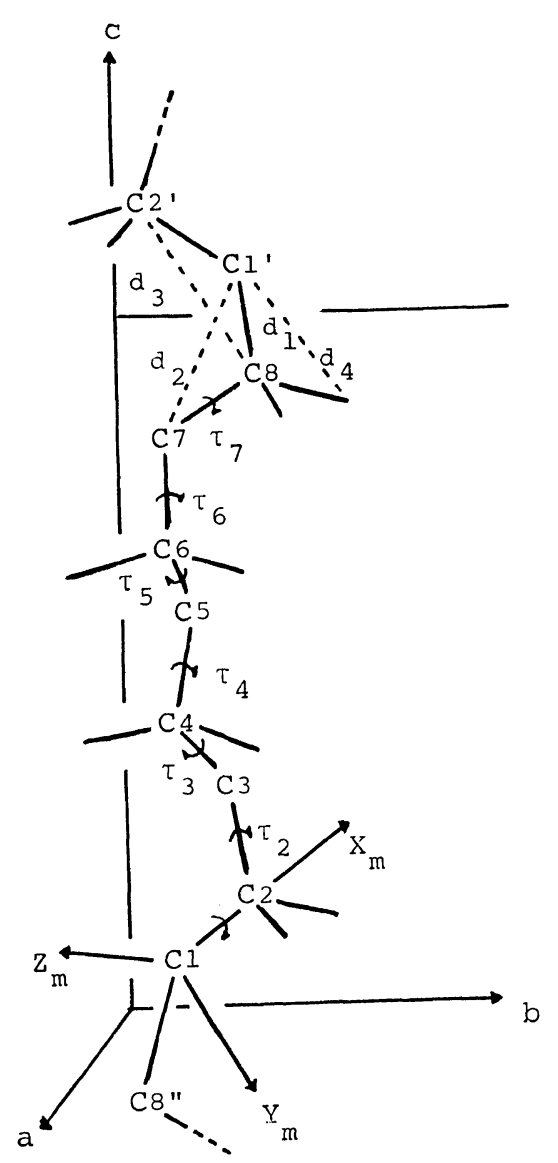

Figure 4. Parameters and constraining conditions used for calculation of PIB. The dihedral angle $Y_{\mathrm{m}}-\mathrm{C} 1-\mathrm{C} 2-\mathrm{C} 3$ is $-163^{\circ}$.

three Eulerian angles $\theta, \phi, \chi$, and six internal rotation angles $\tau_{2}, \tau_{3}, \tau_{4}, \tau_{5}, \tau_{6}$, and $\tau_{7}$ as shown in Figure 4. The distances to be fixed for keeping the bond length and bond angles between successive asymmetric units are $d_{1}(=1.54 \mathrm{~A})$, 
Table IV. Stable crystal structure model of PIB

\begin{tabular}{|c|c|c|c|}
\hline \multicolumn{2}{|l|}{ Model (helical conformation) } & $\begin{array}{c}\text { Start } \\
\text { (uniform) }\end{array}$ & $\begin{array}{c}\text { Final } \\
\text { (deformed) }\end{array}$ \\
\hline \multicolumn{4}{|c|}{ Packing energy, $\mathrm{kcal} / \mathrm{mol}$ of asym. unit } \\
\hline \multicolumn{2}{|l|}{$E_{\mathrm{a}}$ (intramolecular) } & 20.1 & 20.6 \\
\hline \multicolumn{2}{|l|}{$E_{\mathrm{r}}$ (intermolecular) } & 3.5 & 0.3 \\
\hline \multicolumn{2}{|l|}{$E($ total) } & 23.6 & 20.9 \\
\hline \multicolumn{4}{|l|}{ Parameters $^{\mathrm{a}}$} \\
\hline \multirow{3}{*}{$\begin{array}{l}\text { Fractional coordinates } \\
\text { of the } C(1) \text { atom }\end{array}$} & $x$ & 0.243 & 0.219 \\
\hline & & -0.044 & -0.035 \\
\hline & & $\begin{array}{r}0.032 \\
101.6^{\circ}\end{array}$ & $\begin{aligned} 0.013 \\
999^{\circ}\end{aligned}$ \\
\hline \multirow[t]{2}{*}{ Eulerian angles $^{\mathrm{b}}$} & $\begin{array}{c}\theta \\
\phi\end{array}$ & $\begin{array}{l}101.6^{\circ} \\
149.4^{\circ}\end{array}$ & $\begin{array}{r}99.9^{\circ} \\
129.0^{\circ}\end{array}$ \\
\hline & $\chi$ & $-123.0^{\circ}$ & $-117.9^{\circ}$ \\
\hline \multirow[t]{5}{*}{ Internal rotation angles ${ }^{c}$} & $\begin{array}{l}\lambda \\
\tau_{2}\end{array}$ & $-163.0^{\circ}$ & $-159.4^{\circ}$ \\
\hline & $\tau_{3}$ & $-54.4^{\circ}$ & $-55.0^{\circ}$ \\
\hline & $\tau_{4}$ & $\begin{aligned}-163.0^{\circ} \\
-54.4^{\circ}\end{aligned}$ & $\begin{array}{r}-160.6^{\circ} \\
-569^{\circ}\end{array}$ \\
\hline & $\tau_{5}$ & $\begin{aligned}-54.4 \\
-163.0^{\circ}\end{aligned}$ & $\begin{aligned}-140.9 \\
-14.5^{\circ}\end{aligned}$ \\
\hline & $\tau_{7}$ & $-54.4^{\circ}$ & $-50.1^{\circ}$ \\
\hline
\end{tabular}

a Number of independent parameters $=$ (parameters) - (constraining conditions) $=12-4=8$, corresponding to total number of two molecular position parameters $z_{\mathrm{mol}}, \phi_{\mathrm{mol}}$, and 6 internal rotation angles.

b The $Y_{\mathrm{m}}$ axis was taken in the plane defined by the atoms $\mathrm{C}^{\prime \prime}-\mathrm{C} 1-\mathrm{C} 2$, resulting in the dihedral angle $Y_{\mathrm{m}}-\mathrm{C} 1-\mathrm{C} 2-\mathrm{C} 3=-163^{\circ}$ (see Figure 4).

c The final internal rotation angles $\tau_{1}=-66.1^{\circ}$ and $\tau_{8}=-165.2^{\circ}$ were calculated from the final coordinates in the minimization.

(a)

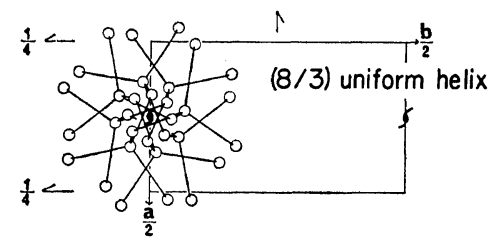

(b)

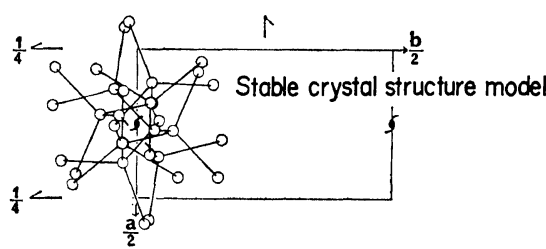

(c)

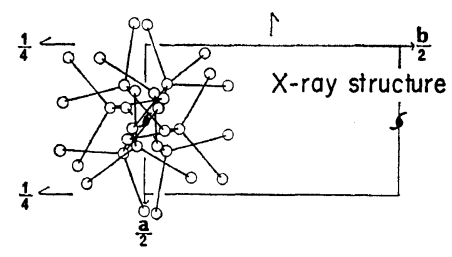

Figure 5. Crystal structure models of PIB: (a), starting uniform helical model; (b), stable crystal structure model obtained by the energy minimization; (c), the structure determined by $\mathrm{X}$-ray analysis. ${ }^{15}$
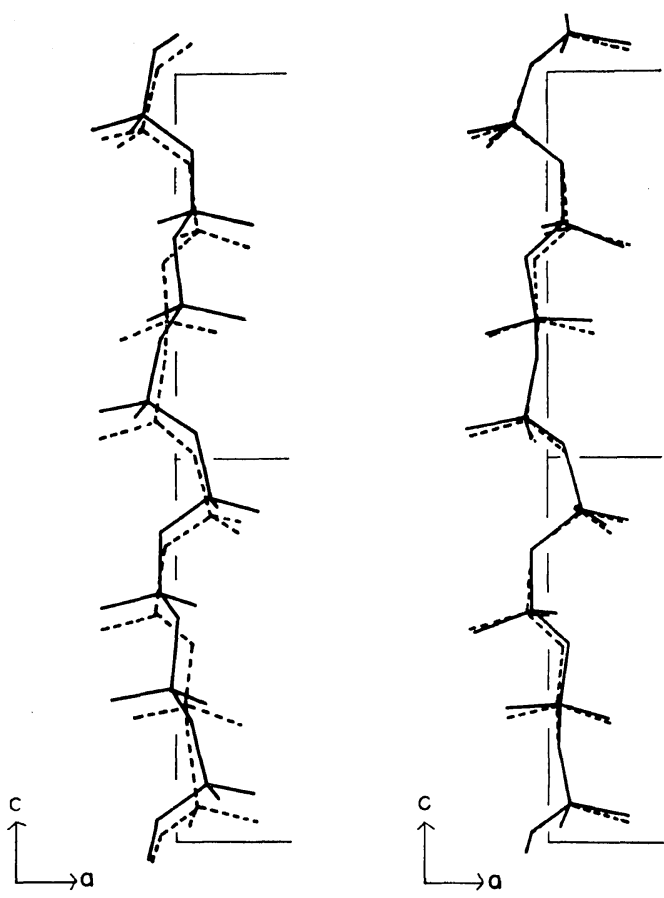

(a)

(b)

Figure 6. Solid lines indicate (a) starting uniform helical model and (b) stable crystal structure model obtained by the energy minimization of PIB. Broken line indicates the structure determined by $\mathrm{X}$-ray analysis.

Table V. Stable crystal structure models calculated by the energy minimization method

\begin{tabular}{|c|c|c|c|c|c|c|c|}
\hline \multirow[t]{2}{*}{$\begin{array}{l}\text { Poly- } \\
\text { mer }\end{array}$} & \multicolumn{3}{|c|}{$\begin{array}{l}\text { Packing energy, }{ }^{a} \\
\mathrm{kcal} / \mathrm{mol} \text { of asym- } \\
\text { metric unit }\end{array}$} & \multicolumn{4}{|c|}{$\begin{array}{c}\text { Average deviation } \bar{d} \text { from } \\
\text { the atomic coordinates } \\
\text { determined by X-ray } \\
\text { analysis } \text {, A }\end{array}$} \\
\hline & $E_{\mathrm{a}}$ & $E_{\mathrm{r}}$ & $E$ & $\bar{d}$ & $\overline{d_{x}}$ & $\overline{\boldsymbol{d}}_{y}$ & $\overline{d_{z}}$ \\
\hline PIB & $\begin{array}{c}20.6 \\
(16.8)\end{array}$ & $\begin{array}{r}0.3 \\
=(2.4)\end{array}$ & $\begin{array}{c}20.9 \\
(19.2)\end{array}$ & 0.11 & 0.09 & 0.09 & 0.12 \\
\hline PEO & $\begin{array}{c}1.9 \\
(0.7)\end{array}$ & $\begin{array}{r}7.5 \\
(13.0)\end{array}$ & $\begin{array}{c}9.4 \\
(13.7)\end{array}$ & 0.23 & 0.24 & 0.13 & 0.20 \\
\hline PP & $\begin{array}{c}4.2 \\
(4.2)\end{array}$ & $\begin{array}{r}0.1 \\
(0.2)\end{array}$ & $\begin{array}{c}4.3 \\
(4.4)\end{array}$ & 0.10 & 0.07 & 0.05 & 0.17 \\
\hline
\end{tabular}

a $E=E_{\mathrm{a}}$ (intramolecular) $+E_{\mathrm{r}}$ (intermolecular).

b $\bar{d}$ was calculated by using eq 9 .

c Parentheses indicate the values with the structures determined by $\mathrm{X}$-ray analyses.

$d_{2}(=2.523 \mathrm{~A}), d_{3}(=2.768 \mathrm{~A})$, and $d_{4}(=2.515 \mathrm{~A})$. The rough crystal structure model composed of the $(8 / 3)$ uniform helices (obtained from model $I$ in ref 15) was used as the starting model of the minimization. After 9 minimization cycles, the sum of intra- and intermolecular repulsive 
energies, $E$, was reduced from $23.6 \mathrm{kcal} / \mathrm{mol}$ of asymmetric unit to $20.9 \mathrm{kcal} / \mathrm{mol}$, and the molecular conformation deviated from the uniform helix (Table IV). The starting and final crystal structure models are shown in Figures 5 and 6, and compared with the structure determined by $\mathrm{X}$-ray analysis. The energetically stable crystal structure model obtained by the present energy minimization method gave a close agreement $(\bar{d}=$ $0.11 \mathrm{~A}$ ) with that determined by X-ray analysis, as shown in Table $V$. This fact indicates that the appreciable deformation from the $(8 / 3)$ uniform helix is energetically favorable to dense molecular packing into the orthorhombic unit cell.

Poly(ethylene oxide)

Since seven monomeric units in the fiber identity period form the crystallographic asymmetric unit, the $(7 / 2)$ uniform helical symmetry is not necessarily kept in the crystal. The

(a)

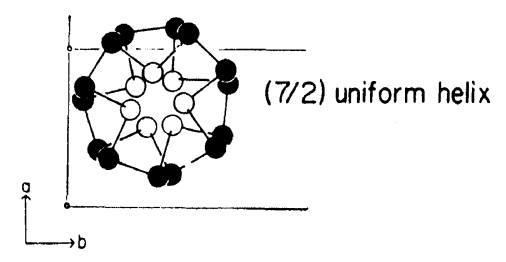

(b)

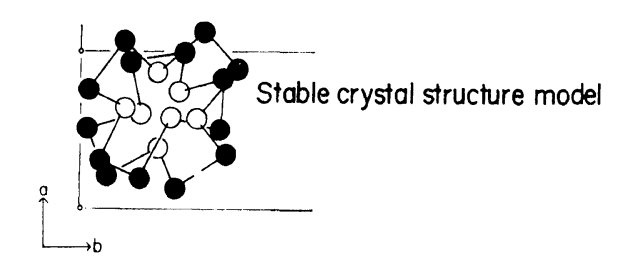

(c)

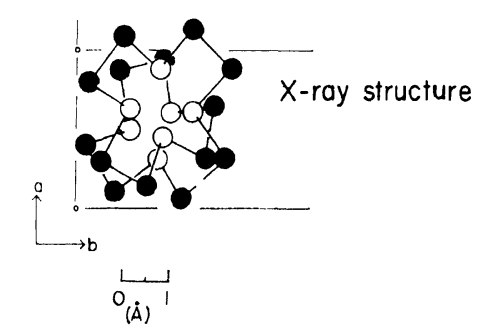

Figure 7. Crystal structure models of PEO: (a), starting uniform helical model; (b), stable crystal structure model obtained by the energy minimization; (c), the structure determined by X-ray analysis. ${ }^{16}$ crystal structure model composed of the (7/2) uniform helices was used as the starting model, and the packing energy $E$ reduced from 35.2 $\mathrm{kcal} / \mathrm{mol}$ of asymmetric unit to $9.4 \mathrm{kcal} / \mathrm{mol}$ after the minimization. The stable crystal structure model gave a fairly close agreement $(\bar{d}=0.23 \mathrm{~A})$ with the structure determined by X-ray analysis as shown in Figure 7. In the case of PEO, considerable distortion from the (7/2) uniform helical symmetry may be expected, since the molecular chain is more flexible because there are no side groups and the intermolecular forces act on the neighboring skeletal chain atoms more directly. ${ }^{16}$ The present result clarified this from the viewpoint of the energy calculation.

Isotactic Polypropylene

It is known that the IPP crystal contains four $(3 / 1)$ helical chains in the monoclinic unit cell. ${ }^{17-19}$ The unit cell, however, does not have $3_{1}$ symmetry at the molecular positions, so that the chain does not necessarily have the uniform
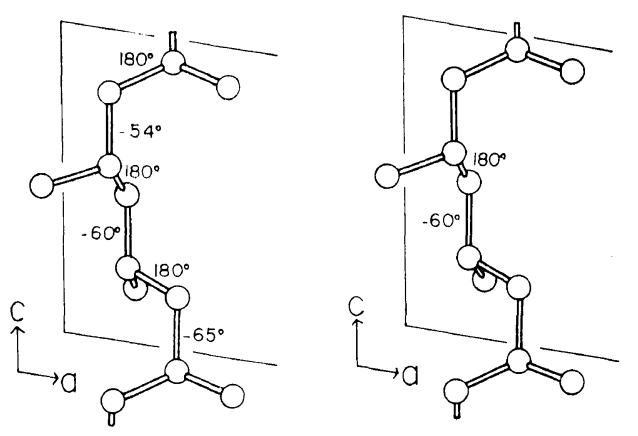

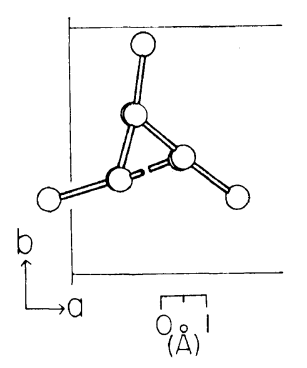

Stable crystal structure model

(a)

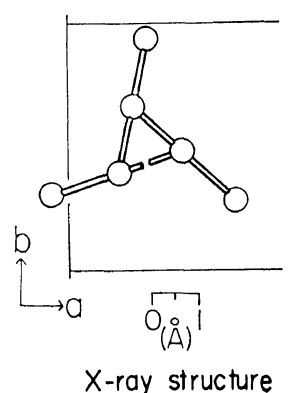

(b)
Figure 8. Crystal structure models of IPP: (a), stable crystal structure model obtained by the energy minimization; (b), the structure determined by X-ray analysis. ${ }^{18}$ 
helical symmetry. The stable crystal structure model calculated under the non-uniform helix symmetry, however, did not deviate appreciably from the uniform helix, and the packing energy $E$ showed almost no reduction. These models, starting and final, are quite similar to the structure determined by X-ray analysis $(\bar{d}=0.11$ and $0.10 \mathrm{~A}$, respectively). Figure 8 shows the stable crystal structure model and the structure by $\mathrm{X}$ ray analysis. The intermolecular interactions are not the dominant factor to define the molecular conformation in this case.

\section{Isotactic Poly(4-methyl-1-pentene)}

The unit cell of poly(4-methyl-1-pentene) (P4MP) is tetragonal, $\mathrm{P} \overline{4} \mathrm{~b} 2\left(\mathrm{D}_{2 \mathrm{~d}}^{7}\right)$ with $a=18.68 \mathrm{~A}$ and $c$ (fiber axis) $=13.69 \mathrm{~A}$, and four $(7 / 2)$ helical chains pass through the unit cell. However, the $(7 / 2)$ helical symmetry does not exist at the molecular positions in the tetragonal cell, so that the $(7 / 2)$ uniform helix is not necessarily kept in the crystal. Furthermore, the appearance of 006 and 008 reflections on the meridian of the $\mathrm{X}$-ray photograph suggests that the molecular conformation deviates from the exact (7/2) uniform helix. The refinement of the crystal structure model of P4MP is difficult by using the ordinary least-squares method in terms of diffraction data alone, because of the large number of parameters, 29 , in comparison with the number of the intensity data, 66 . The refinement gave a small value of the discrepancy factor $R\left(=\sum\left|\sqrt{I_{\mathrm{o}}}-\sqrt{I_{\mathrm{c}}}\right| / \sum \sqrt{I_{\mathrm{o}}}\right)$, but the resultant molecular conformation deformed unreasonably from the (7/2) helix and there appeared many too close interatomic distances between the neighboring molecular chains.

Therefore, the newly derived least-squares method of eq 8 was applied to the refinement of the structure model of P4MP. The crystal was assumed to have a statistically disordered structure consisting of a random mixture of upward and downward helices of $1: 1$ ratio. Therefore, the contribution of the upward and downward helices with a probability of one half weight was taken into account in the energy calculation. The value of $\omega$ was assumed to be 0.1 in this refinement. The refinement, starting from the $(7 / 2)$ uniform helix model, gave a good convergence of the discrepancy
Table VI. Refinement of the crystal structure model of P4MP by the constrained leastsquares method with energy constraint

\begin{tabular}{llcc}
\hline & & Start & Final \\
\hline$R, \mathrm{a} \%$ & & 25 & 20 \\
$E,{ }^{\mathrm{b}} \mathrm{kcal} / \mathrm{mol}$ of asym. & & 163.9 & 30.4 \\
Intermolecular & $\mathrm{CH}_{3} \cdots \mathrm{CH}_{3}$ & 3.25 & 3.73 \\
closest & $\mathrm{CH}_{3} \cdots \mathrm{CH}_{2}$ & 3.96 & 3.99 \\
distance, $\mathrm{A}$ & $\mathrm{CH}_{3} \cdots \mathrm{CH}$ & 3.51 & 3.73 \\
\hline
\end{tabular}

a Discrepancy factor.

b Packing energy.

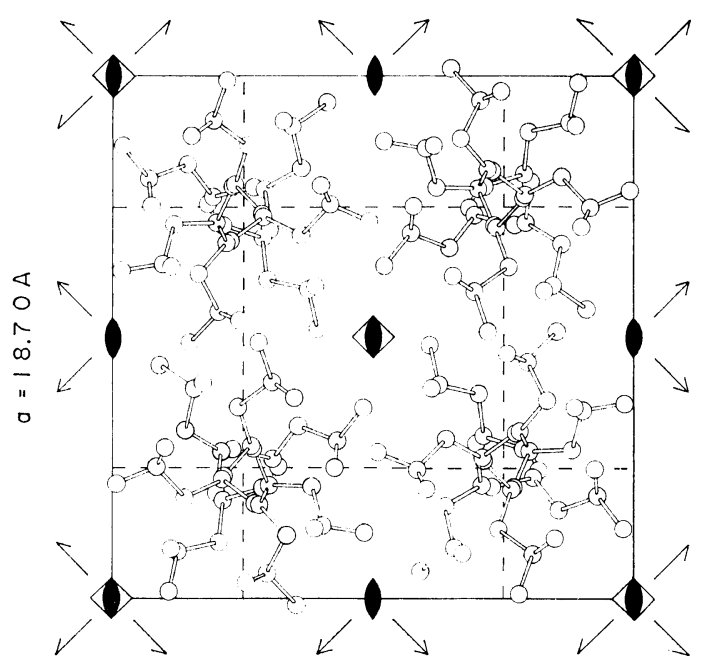

Figure 9. Crystal structure of P4MP. Only the upward helices are illustrated for simplicity.

factor $(R=20 \%)$ and the packing energy $(E=30.4$ $\mathrm{kcal} / \mathrm{mol}$ of asymmetric unit), as shown in Table VI. The final crystal structure model thus obtained is shown in Figure 9. There are no unreasonably short interatomic distances. The result of the detailed structure analysis of P4MP will be published elsewhere. ${ }^{21}$

\section{DISCUSSION}

In spite of the simplified treatment which takes account of the repulsive nonbonded interactions, close agreement were obtained between the calculated stable structure models and the structures determined by X-ray analyses for PIB, PEO, and IPP (Table V). The reasons may be as follows. (a) In this minimization method the repulsive energy was calculated within the 


\section{H. Kusanagi, H. Tadokoro, and Y. Chatani}

unit cell determined by the X-ray diffraction method. This means the attractive forces are implicitly taken into account. In other words, the use of observed cell dimensions effectively incorporates molecular attractions into the energy minimization, since the molecular volumes are restricted. The minimum repulsive energy structure corresponds to the "closest packing" geometry under the condition of a packing density ${ }^{22,23}$ given by the cell dimensions and the number of molecules in the unit cell. (b) The packing energy calculation may be favorable for polymer crystals, in comparison with low-molecularweight compounds, because there is no rotational freedom about the axes perpendicular to the chain direction, since the polymer chains are parallel in the crystallite.

In principle, the stability of polymer crystals must be compared in terms of free energy. The contribution of the normal vibration entropy to the free energy of polyethylene was calculated in this laboratory, ${ }^{24}$ and it was found that orthorhombic crystal is more stable than monoclinic crystal. For the cases of PIB, PEO, and IPP, however, enormous calculations will be needed to take account for the contribution of vibrational entropy to the calculations.

Finally, the present energy minimization method may be useful in the following ways: (a) the setting up of more suitable crystal structure models at the early stage of the process of $\mathrm{X}$-ray analysis, and (b) for obtaining more reasonable molecular conformation and packing geometry in the least-squares refinement of the structure factors.

After completion of the present work, the authors were advised that Arnott, et al.,${ }^{25}$ has independently derived a least-squares method with energy constraint essentially similar to the one given here.

\section{REFERENCES}

1. H. Sakahihara-Kitahama and H. Tadokoro, $J$. Macromol. Sci., Phys., 9, 511 (1974).
2. M. Yokouchi, H. Tadokoro, and Y. Chatani, Macromolecules, 7, 769 (1974).

3. H. Sakakihara, Y. Takahashi, H. Tadokoro, N. Oguni, and H. Tani, ibid., 6, 205 (1973).

4. M. Yokouchi, Y. Chatani, H. Tadokoro, and H. Tani, Polym. J., 6, 248 (1974).

5. H. Kusanagi, H. Tadokoro, Y. Chatani, and K. Suehiro, Macromolecules, in press.

6. R. F. McGuire, G. Vanderkooi, F. A. Momany, R. T. Ingwall, G. M. Crippen, N. Lotan, R. W. Tuttle, K. L. Kashuba, and H. A. Scheraga, ibid., 4, 112 (1971).

7. J. Hermans, Jr., and J. E. McQueen, Jr., Acta Crystallogr., A30, 730 (1974).

8. D. E. Williams, ibid., A25, 464 (1969).

9. K. Tai, Doctoral Thesis, Osaka University, Toyonaka, Osaka, 1974.

10. R. L. McCullough and P. E. McMahon, Trans. Faraday Soc., 60, 2089 (1964).

11. S. Arnott and A. J. Wonacott, Polymer, 7, 157 (1966).

12. Y. Takahashi, T. Sato, H. Tadokoro, and Y. Tanaka, J. Polym. Sci., Polym. Phys. Ed., 11, 233 (1973).

13. C. Scheringer, Acta Crystallogr., 16, 546 (1963).

14. H. Sugeta and T. Miyazawa, Biopolymers, 5, 673 (1967).

15. T. Tanaka, Y. Chatani, and H. Tadokoro, $J$. Polym. Sci., Polym. Phys. Ed., 12, 515 (1974).

16. Y. Takahashi and H. Tadokoro, Macromolecules, 6, 672 (1973).

17. G. Natta and P. Corradini, Nuovo Cimento Suppl., 15, 40 (1960).

18. Z. Mencik, J. Macromol. Sci., Phys., 6, 101 (1972).

19. M. Hikosaka and T. Seto, Polym. J., 5, 111 (1973).

20. P. Zugenmaier and A. Sarko, Acta Crystallogr., B28, 3158 (1972).

21. H. Kusanagi, M. Takase, Y. Chatani, and H. Tadokoro, to be published.

22. A. I. Kitaigorodsky, "Molecular Crystals and Molecules," Academic Press, New York, N.Y., 1973.

23. V. P. Privalko, Polym. J., 7, 202 (1975).

24. M. Kobayashi and H. Tadokoro, Macromolecules, 8, 897 (1975).

25. S. Arnott, W. E. Scott, E. A. Rees, and C. G. McNab, J. Mol. Biol., 90, 253 (1974). 